\title{
IncRNA SNHG15 Induced by SOX12 Promotes the Tumorigenic Properties and Chemoresistance in Cervical Cancer via the miR- 4735-3p/HIF1a Pathway
}

\author{
Jiang Yang, Mei Yang, Huabing Lv, Min Zhou, Xiaogang Mao, Xiaomin Qin, Ying Xu, \\ Lin Li $\mathbb{D}$, and Hui Xing \\ Department of Obstetrics and Gynecology, Xiangyang Central Hospital, Affiliated Hospital of Hubei University of Arts and Science, \\ Xiangyang, Hubei, China \\ Correspondence should be addressed to Lin Li; 11119@tom.com and Hui Xing; huixinghx123@163.com
}

Received 31 July 2021; Revised 2 November 2021; Accepted 8 November 2021; Published 12 January 2022

Academic Editor: Peichao CHEN

Copyright (c) 2022 Jiang Yang et al. This is an open access article distributed under the Creative Commons Attribution License, which permits unrestricted use, distribution, and reproduction in any medium, provided the original work is properly cited.

Cervical cancer (CC) is one of the most common malignancies in females, with high prevalence and mortality globally. Despite advances in diagnosis and therapeutic strategies developed in recent years, CC is still a major health burden worldwide. The molecular mechanisms underlying the development of CC need to be understood. In this study, we aimed to demonstrate the role of lncRNA SNHG15 in CC progression. Using qRT-PCR, we determined that lncRNA SNHG15 is highly expressed in CC tumor tissues and cells. IncRNA SNHG15 knockdown also reduces the tumorigenic properties of CC in vitro, as determined using the MTT, EdU, flow cytometry, and transwell assays. Using bioinformatics analysis, RNA pull-down, ChIP, and luciferase reporter assays, we verified the molecular mechanisms of lncRNA SNHG15 in CC progression and found that lncRNA SNHG15 expression in CC cells is transcriptionally regulated by SOX12; moreover, lncRNA SNHG15 promotes CC progression via the miR-4735-3p/HIF1a axis. This study can provide a potential target for CC diagnosis or therapeutic strategies in the future.

\section{Introduction}

Cervical cancer (CC) ranks next to breast cancer as the most common malignancy and is the third leading cause of cancer-related deaths in females globally [1]. The health burden associated with CC is more severe in developing countries [2], particularly in low-income countries, than in developed countries [2]. Multiple risk factors contribute to the initiation and progression of CC, such as viral infection, genetic influences, and human papillomavirus (HR-HPV) genotype infection $[3,4]$. Despite improvement in clinical intervention strategies, such as the global application of standard vaccination, surgical resection innovation, and periodic cancer screening in recent decades, the outcomes of patients with CC remain poor. Therefore, the molecular mechanisms underlying CC development need to be understood, and novel therapeutic targets for the prevention and treatment of CC have to be urgently developed.
Long noncoding RNAs (lncRNAs) are newly discovered no-coding RNAs longer 200 nt [5]. Emerging evidence has elucidated the crucial role of lncRNAs in various diseases, including cancers. lncRNA NBR2 suppresses tumorigenesis in hepatocellular cancer via modulating autophagy level [6]. IncRNA GATA3-AS1 regulates triple-negative breast cancer by facilitating tumorigenesis and immune escape phenomena [7]. IncRNA HOTAIR facilitates exosome secretion in hepatocellular cancer by regulating RAB35 and SNAP23 [8]. IncRNA ASB16-AS1 plays an oncogenic role in renal cell carcinoma progression by acting as a ceRNA for miR-185-5p/miR-214-3p [9]. The lncRNA small nucleolar RNA host gene 15 (lncRNA SNHG15) is located on chromosome $7 \mathrm{p} 13$ and was first elucidated in a study of cellular stress responses $[10,11]$. The function of lncRNA SNHG15 in various cancers has also been investigated, including breast cancer, colorectal cancer, gastric cancer, hepatocellular cancer, and lung cancer [12-16]. Meanwhile, whether 
lncRNA SNHG15 participates in CC progression remains unclear.

In this study, we aimed to demonstrate the role of lncRNA SNHG15 in CC progression. We found that lncRNA SNHG15 was abundantly expressed in CC tumors and cells. lncRNA SNHG15 knockdown significantly attenuated tumorigenesis in CC in vitro and in vivo, as determined using the MTT, EdU, flow cytometry, and transwell assays, in addition to animal experiments. Further, our study revealed that lncRNA SNHG15 influenced the chemoresistance of CC cells to cisplatin. We then investigated the molecular mechanisms of lncRNA SNHG15 in CC cells by conducting bioinformatics analysis, RNA pull-down, ChIP, and luciferase reporter assay. It was found that lncRNA SNHG15 expression in CC cells was transcriptionally regulated by SRY-Box Transcription Factor 12 (SOX12). Further, we found that lncRNA SNHG15 upregulated HIF1a expression to regulate tumorigenesis in CC by sponging miR-4735$3 p$. Thus, our study elucidated the effect of SOX12/lncRNA SNHG15/miR-4735-3p/HIF1a axis on the biological features of CC.

\section{Materials and Methods}

2.1. Clinical Samples. A total of twenty-eight pairs of CC tumor tissues and comparative normal tissues were collected from Xiangyang Central Hospital within the period 2019/06 to $2020 / 06$. All CC tissues were immediately stored in $-80^{\circ} \mathrm{C}$ liquid nitrogen until the experiment was conducted. Informed consent was obtained from each patient. All CC tissues were confirmed by two pathologists, independently. This study was performed following the principles of the Declaration of Helsinki and was approved by the ethical committee of Xiangyang Central Hospital.

2.2. Cell Culture and Transfection. All cervical cancer cell lines (SiHa, HeLa, Caski, C-33A, and MS751) and normal cell line HEKn were commercially obtained from the Committee on Type Culture Collection of the Chinese Academy of Sciences (Shanghai, China). Cells were cultured using Dulbecco's Modified Eagle's Medium (DMEM; Invitrogen, USA) supplied with $10 \%$ fetal bovine serum (FBS) at $37^{\circ} \mathrm{C}$ in a humidified $5 \% \mathrm{CO}_{2}$ environment.

All siRNAs targeting lncRNA SNHG15 were synthesized and obtained from Sigma-Aldrich (USA). Moreover, miRNA mimics, inhibitors, and normal controls were procured from GenePharma (Shanghai, China). The pcDNA3.1 (+) vector (GenePharma, Shanghai) was applied to generate overexpression vectors. The siRNA sequences are as follows: si-NC: F: $5^{\prime}$-CAGUCGCGUUUGCGACUGGC- $3^{\prime}$ and R: $5^{\prime}$ -GCCAGUCGCAAACGCGACUG-3', si-SNHG15\#1: F: $5^{\prime}$ CCUUGAGUCUCAUGUUCAA- $3^{\prime}$ and R: $5^{\prime}$-UUGAAC AUGAGACUCAAGG-3' ${ }^{\prime}$, si-SNHG15\#2: F: 5'-GAGCUU ACUGUCACAGCAA- $3^{\prime}$ and R: $5^{\prime}$-UUGCUGUGACA GUAAGCUC-3'. A Lipofectamine 3000 (Invitrogen) was applied to conduct transfections.

2.3. RNA Extraction and $q R T-P C R$. All RNAs were collected from tissues and cells by using the TRIzol Reagent (Thermo
Fisher Scientific) in accordance with the manufacturer protocols. Reverse-transcription was performed using the SuperScript ${ }^{\mathrm{TM}}$ III First-Strand Synthesis System (Thermo Fisher Scientific). The expression level of the indicated gene was measured using the SYBR Green Real-Time PCR Master Mixes (Thermo Fisher Scientific) on the ABI Prism 700 thermal cycler (Applied Biosystems, Foster City, CA). U6 and GAPDH were used as internal controls. The relative gene expression was calculated using the $2-\Delta \Delta \mathrm{Ct}$ method. The primers applied in this study are as follows: lncRNA SNHG15: F: GCTGAGGTGACGGTCTCAAA and R: GCCTCCCAGTTTCATGGACA, miR-4735-3p: F: $5^{\prime}$ GGTAGCTGAGAACATTACAG- $3^{\prime}$ and R: $5^{\prime}$-CTATTC TGGAACATCAAGCC- $3^{\prime}$, HIF1a: F: $5^{\prime}$-TGTGAACCCAT TCCTCATCCA-3' and R: 5'-GGCTCATAACCCATCA ACTCA-3', SOX12: F: 5' -CGCGATGGTGCAGCAGCG$3^{\prime}$ and R: 5'-GCCACTGGTCCATGATCTTC-3', U6: F: TCCGATCGTGAAGCGTTC and R: GTGCAGGGTCC GAGGT, GAPDH: F: CGGAGTCAACGGATTTGGTCG TAT and R: AGCCTTCTCCATGGTGGTGAAGAC.

2.4. Western Blot. All proteins were isolated from tissues and cells by using the RIPA Buffer (Solarbio, R0020). Protein samples were maintained by SDS-PAGE using $8 \%-12 \%$ gels (Beyotime, P0012A). Subsequently, protein samples were then electronically transferred onto a polyvinylidene difluoride membrane $(0.45 \mu \mathrm{m})$ (Millipore, IPVH00010) and then blocked using $5 \%$ skimmed milk. Next, primary antibodies were subsequently used to incubate with membranes at $4^{\circ} \mathrm{C}$ for 12 hours, followed by incubation with secondary antibodies at room temperature for 2 hours. Results were visualized using the UVP ChemiDoc-It Imaging System (UVP, CA, USA). The antibodies used in this study were as follows: HIF1a (1:1000; ab237544; Abcam) and GAPDH (1:1000; \# 5174S; CST).

2.5. Cell Proliferation Detection. 3-(4,5-Dimethylthiazol-2yl)-2,5-diphenyltetrazolium bromide (MTT) assay. Cells were cultured in a 96 well-plate for proliferation detection. The culture medium was added sequentially with $10 \mu \mathrm{L}$ MTT (Invitrogen) for 24, 48, and $72 \mathrm{~h}$. Formazan was dissolved using DMSO (Sigma, USA). After $4 \mathrm{~h}$, the absorbance of each well was measured on a plate reader at $570 \mathrm{~nm}$. The experiment was conducted in triplicate.

5-Ethynyl-2 ' -deoxyuridine (EdU) experiment. The experimental cells were first cultured in a 96-well plate after transfection. The EdU kit (KeyGEN BioTECH) was used to stain cells in the well in accordance with instructions provided by the manufacturer. The results (EdU-positive cells) were recorded by a fluorescence microscopy.

2.6. Cell Apoptosis Detection. The apoptosis rate of infected CC cells was detected by flow cytometry analysis. The infected CC cells were stained using an Annexin Vfluorescein isothiocyanate (FITC)/propidium iodide (PI) kit (BD Biosciences, USA), and the results were calculated with a flow cytometer using the CellQuest software version 0.9.3.1 (BD Biosciences, USA). 


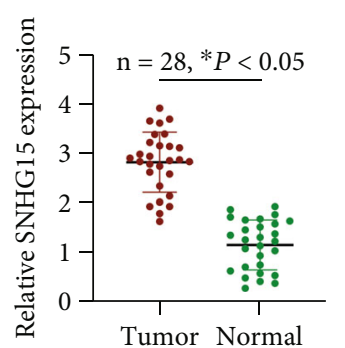

(a)

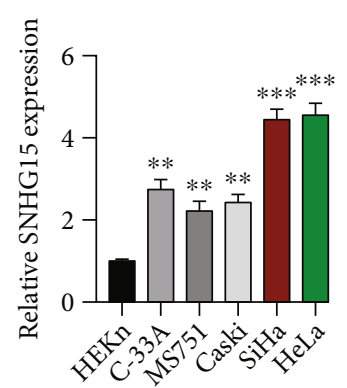

(b)

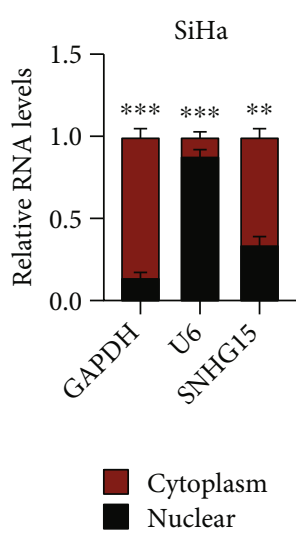

(c)

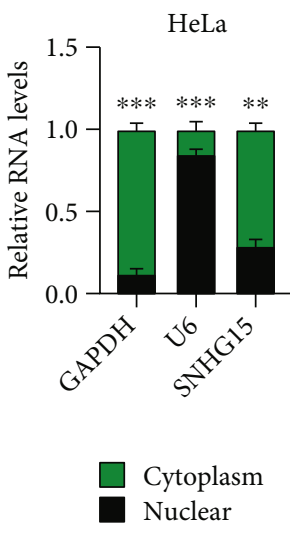

(d)

FiguRe 1: Expression characteristics of lncRNA SNHG15 in cervical cancer (CC). (a) lncRNA SNHG15 expression in CC tumor samples $(n=28)$ and adjacent normal samples $(n=28)$, measured by qRT-PCR. (b) lncRNA SNHG15 expression in CC cell lines, assessed by qRT-PCR. (c, d) lncRNA SNHG15 expression distribution in (c) SiHa and (d) HeLa cells, assessed using the cellular distribution assay. Data are presented as mean $\pm \mathrm{SD} ;{ }^{*} p<0.5,{ }^{* *} p<0.01$, and ${ }^{* * *} p<0.001$.

2.7. Cell Migration Detection. The migratory ability of infected CC cells was detected using the transwell assay. In summary, the infected CC cells were seeded in the upper chambers (Costar, USA) supplied with the serum-free RPMI-1640 medium, and the lower chambers without cells were supplied with full medium. After incubation for one day, the migrated cells were fixed with $4 \%$ paraformaldehyde and then stained with crystal violet. The results were recorded by a microscopy.

2.8. Murine Xenograft Assay. SiHa and HeLa cells stably transfected with si-SHNG15\#1, si-SHNG15\#2, or si-NC were injected into mice aged 4 weeks at a density of $1 \times$ $10^{6}$ cells. The dimensions of the tumors were determined, and their weights were recorded after injection for $28 \mathrm{~d}$. The animal procedures conducted in this study complied with the guidelines set by the ethical committee of Xiangyang Central Hospital.

2.9. RNA Pull-down Assay. SiHa and HeLa cells were infected using $50 \mathrm{nM}$ biotinylated probes, which were synthesized and procured from GenePharma (Shanghai, China). After $2 \mathrm{~d}$, the M-280 streptavidin magnetic beads (Sigma-Aldrich, USA) were used to incubate with the cell lysate at $4^{\circ} \mathrm{C}$ for $3 \mathrm{~h}$. The beads were then washed, and the bound RNAs were isolated using the TRIzol Reagent. The results were analyzed by qRT-PCR. The experiment was performed 3 times.

2.10. Luciferase Assays. Cells were first cultured in a 24 -well plate $\left(1 \times 10^{4}\right.$ cells per well $)$ for $2 \mathrm{~d}$. They were then transfected with the indicated vectors using a Lipofectamine 3000 kit (Invitrogen) in accordance with the manufacturer protocols. After $2 \mathrm{~d}$, the luciferase activities were measured using the Dual-Luciferase Reporter Assay System (Promega). The experiment was performed 3 times.

2.11. Chromatin Immunoprecipitation (ChIP) Assay. Infected CC cells were stained with $1 \%$ formaldehyde and lysed at room temperature for $10 \mathrm{~min}$. The genome was frag- mented to $\sim 500$ bp upon sonication. Antibodies were used to incubate the cell lysate for 12 hours. The lysate was subsequently incubated with Protein A Agarose/Salmon Sperm DNA (50\% Slurry) beads for 6 hours. The results were analyzed by qRT-PCR assay.

2.12. Statistical Analysis. The GraphPad Prism software (GraphPad, USA) was used to calculate all data in this study. Statistical analysis was performed using two-tailed Student's $t$-test. $p<0.05$ indicated a significant difference. Data in this study are presented using the mean \pm square deviation $(\mathrm{SD})$.

\section{Results}

3.1. Expression Characteristics of $\operatorname{lncRNA} S N H G 15$ in Cervical Cancer. To identify the possible function of lncRNA SNHG15 in CC progression, we first investigated the expression profile of lncRNA SNHG15 in CC. Twenty-eight pairs of CC tumor tissues and its comparative normal tissues were examined. lncRNA SNHG15 was noticeably upregulated in CC tumor tissues relative to that in normal tissues (Figure 1(a)). We further found that lncRNA SNHG15 was abundantly expressed in CC cell lines (Figure 1(b)) and mainly located in the cell cytoplasm (Figures 1(c) and $1(d))$. On the basis of these combined findings, we hypothesized that lncRNA SNHG15 is involved in CC progression.

3.2. IncRNA SNHG15 Knockdown Attenuates CC Tumorigenesis and Chemoresistance In Vitro. Given the abundant expression of lncRNA SNHG15 in CC tumor tissues and cells, we generated knockdown cell models of lncRNA SNHG15 to explore its cellular function (Figure 2(a)). We first examined the proliferation level of lncRNA SNHG15 knockdown cells by using the MTT assay (Figures 2(b) and 2(c)) and the EdU assay (Figures 2(d) and $2(\mathrm{e})$ ) in SiHa and HeLa cells and found that lncRNA SNHG15 downregulation markedly suppressed CC cell proliferation. IncRNA SNHG15 downregulation significantly promoted the cell apoptosis rate (Figures 2(f) and 2(g)), as 


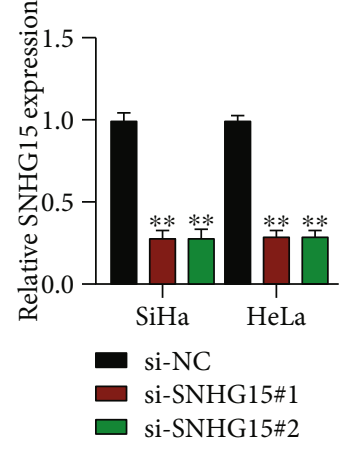

(a)

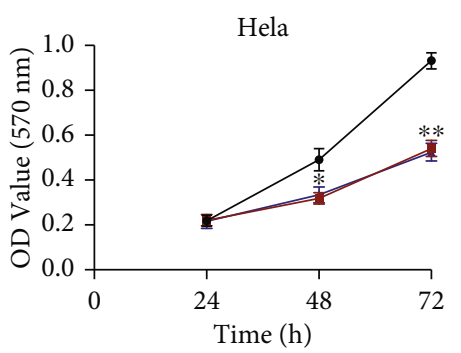

$\rightarrow$ si-NC

- si-SNHG15\#

$\simeq$ si-SNHG15\#2

(c)

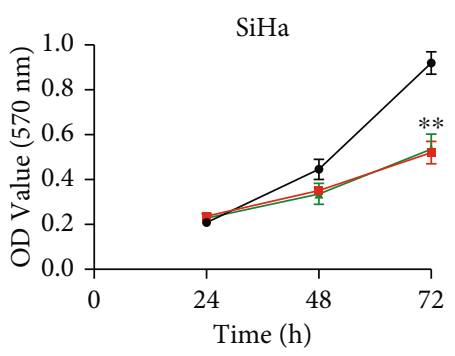

$\rightarrow$ si-NC

- si-SNHG15\#

— si-SNHG15\#2

(b)
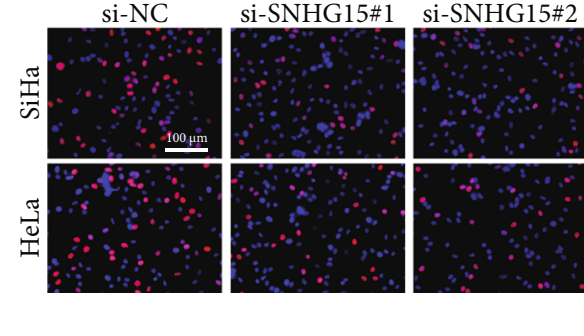

(d)
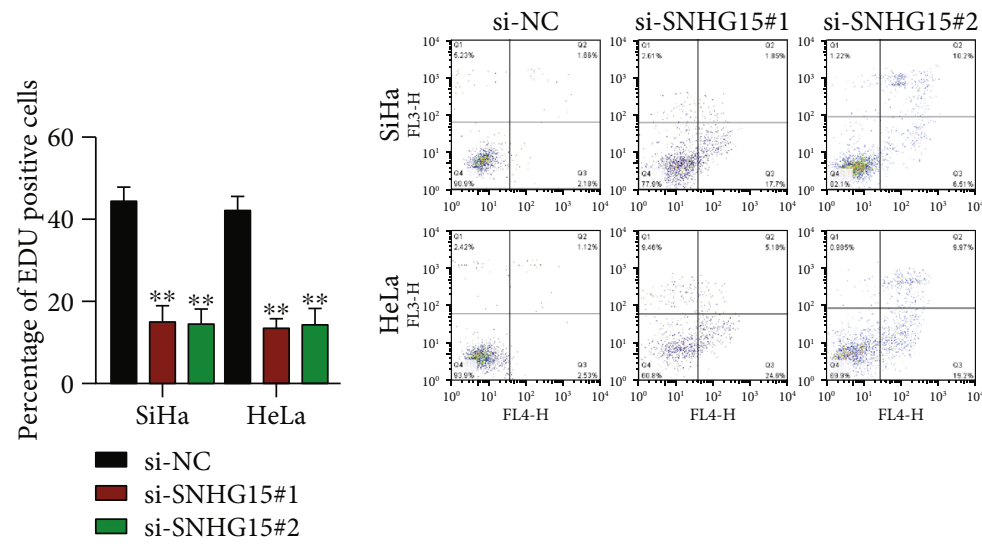

(e)

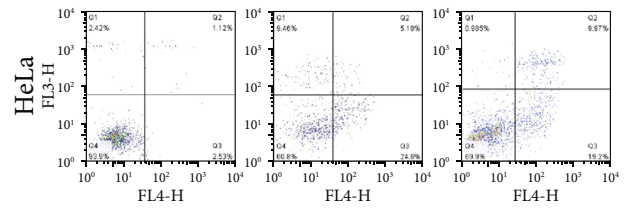

(f)

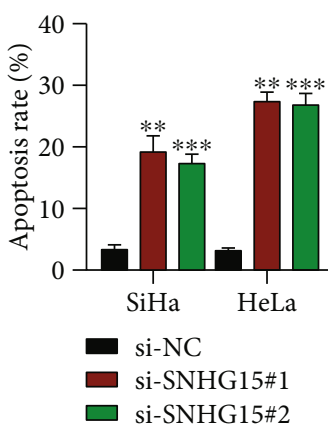

(g)

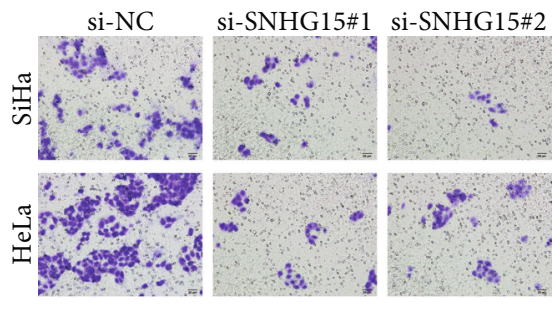

(h)

Figure 2: Continued. 


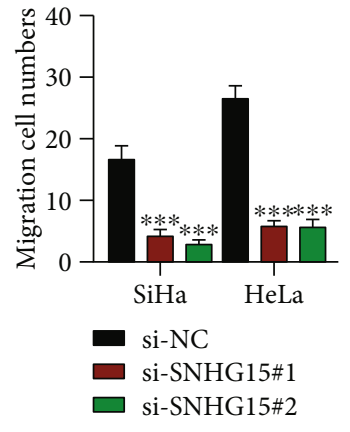

(i)

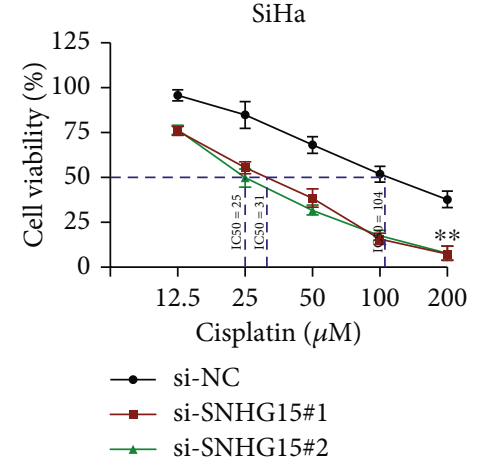

(j)

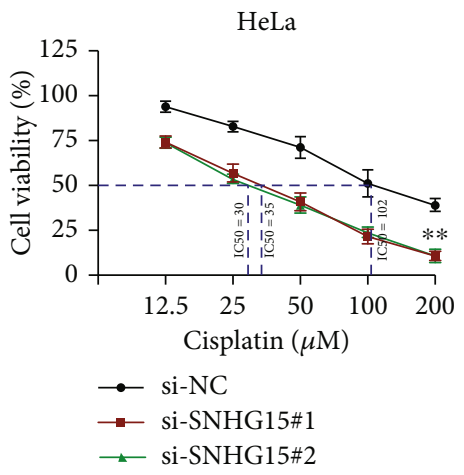

$(\mathrm{k})$

FIGURE 2: IncRNA SNHG15 knockdown attenuates cervical cancer (CC) tumorigenesis and chemoresistance in vitro. (a) lncRNA SNHG15 expression in CC cells after knockdown with two siRNAs, measured by qRT-PCR. (b, c) Cell proliferation in (b) SiHa and (c) HeLa cells, detected using the MTT assay. (d, e) Cell viabilities in (d) SiHa and HeLa cells, evaluated using the EdU assay; (e) analysis of results. (f) Cell apoptosis measured by flow cytometry assay and $(\mathrm{g})$ calculation of the results. (h) Cell migration, assessed using the transwell migration experiment; (i) statistical analysis. (j) $\mathrm{SiHa}$ and $(\mathrm{k}) \mathrm{HeLa}$ cells were treated with cisplatin at varying concentrations; chemoresistance of CC cells was evaluated using the MTT assay. Data are presented as mean $\pm \mathrm{SD} ;{ }^{* *} p<0.01$ and ${ }^{* * *} p<0.001$.

determined by flow cytometer assay. Moreover, lncRNA SNHG15 downregulation distinctly inhibited the CC cell migration, as determined using the transwell assay (Figures 2(h) and 2(i)). Notably, we examined whether lncRNA SNHG15 influences the resistance of CC cells to cisplatin, and lncRNA SNHG15 knockdown reduced the inhibitory concentration $50 \%$ (IC50) of $\mathrm{SiHa}$ and $\mathrm{HeLa}$ cells (Figures $2(\mathrm{j})$ and $2(\mathrm{k})$, respectively), respectively, indicating that lncRNA SNHG15 downregulation reduced the chemoresistance of CC cells to cisplatin. The results demonstrated that lncRNA SNHG15 knockdown inhibited cell proliferation, migration, and chemoresistance to cisplatin, as well as promoted cell apoptosis. Gain-of-function assays were also performed to confirm the functional role of IncRNA SNHG15 in CC progression (Supplementary Materials Figure S1); lncRNA SNHG15 overexpression promoted cell proliferation, migration, and chemoresistance to cisplatin but repressed cell apoptosis.

\subsection{IncRNA SNHG15 Expression in CC Is Transcriptionally} Regulated by SOX12. We investigated the upstream regulator of lncRNA SNHG15 in CC cells. Bioinformatics analysis was conducted, and 6 potential transcriptional regulators were predicted. We found that lncRNA SNHG15 expression was markedly decreased in CC cells under SRY-Box Transcription Factor 12 (SOX12) knockdown (Figures 3(a) and 3(b)) and increased in CC cells upon SOX12 overexpression (Figure 3(c)). The binding motif in SOX12 and the binding sites of the lncRNA SNHG15 promoter were acquired from the JASPAR database (Figures 3(e) and 3(e)). Chromatin immunoprecipitation (ChIP) assay results showed that the IncRNA SNHG15 promoter was pulled down by the SOX12 antibody in the SiHa and HeLa cells (Figure 3(f)). The interaction between SOX12 and the IncRNA SNHG15 promoter was verified by the luciferase reporter assay conducted on the SiHa and HeLa cells (Figures 3(g) and 3(h)). Combined, these findings indicated that SOX12 modulated lncRNA SNHG15 transcription in CC cells.
3.4. IncRNA SNHG15 Sponges miR-4735-3p. The downstream target of lncRNA SNHG15 in CC cells was explored. The prediction from the starBase dataset showed 8 putative miRNA targets. Data from the biotinylated RNA pulldown assay revealed that 4 miRNA targets could potentially interact with lncRNA SNHG15 in CC cells (Figures 4(a) and 4(b)). Among the potential interactions, those between lncRNA SNHG15 and miR-188-5p [17, 18], miR-346 [19], and miR-18b-5p [20] have been previously verified. In the current study, we investigated the relation between lncRNA SNHG15 and miR-4735-3p in CC cells. Results from the RNA pull-down assay indicated that lncRNA SNHG15 could be pulled down by miR-4735-3p probes in SiHa and HeLa cells (Figure 4(c)). Binding sites between lncRNA SNHG15 and miR-4735-3p were revealed (Figure 4(d)), and the transfection efficiency of the miR-4735-3p mimic was assessed (Figure 4(e)). Results from the luciferase reporter assay suggested that lncRNA SNHG15 directly targeted miR-4735-3p in CC cells (Figures 4(f) and 4(g)). Moreover, miR-4735-3p expression in CC cells was upregulated under lncRNA SNHG15 knockdown (Figure 4(h)) and upregulated in CC tumors relative to that in normal samples (Figure 4(i)). These data revealed the miR-4735-3p as a downstream target for lncRNA SNHG15 in CC.

3.5. miR-4735-3p Modulates Tumorigenesis and Chemoresistance in Cervical Cancer. We revealed miR4735-3p as a target for lncRNA SNHG15 in CC cells; however, the function of miR-4735-3p remained unverified. Thus, we conducted a gain- or loss-of-function experiment on miR-4735-3p in CC cells. Cell models were generated as indicated (Figure 5(a)). CC cell proliferation was markedly suppressed by the miR-4735-3p mimic but promoted by the miR-4735-3p inhibitor, as determined using the MTT assay (Figures 5(b)-5(e)) and the EdU assay (Figures 5(f) and 5(g), Supplementary Materials Figures S2A-S2B). Flow cytometry results showed that the CC cell apoptosis rate was promoted by the miR-4735-3p mimic and inhibited by 


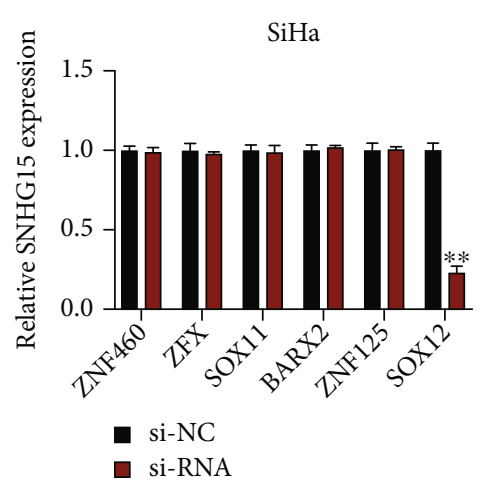

(a)

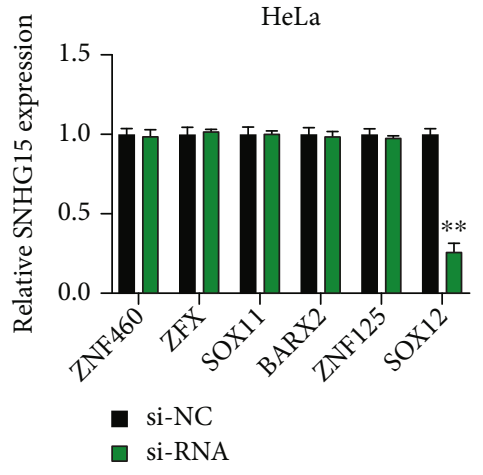

(b)

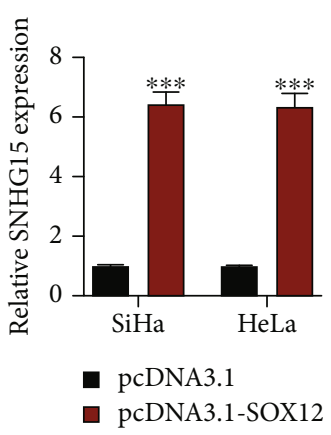

(c)

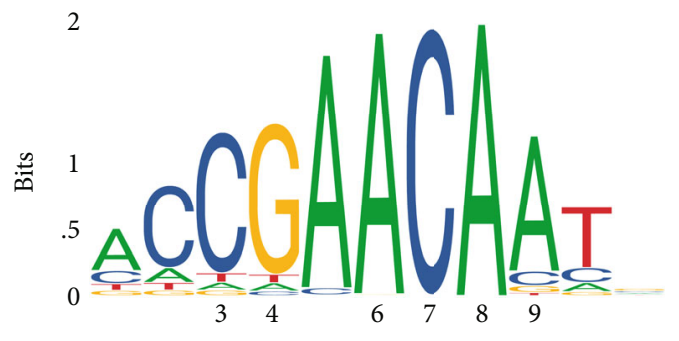

(d)

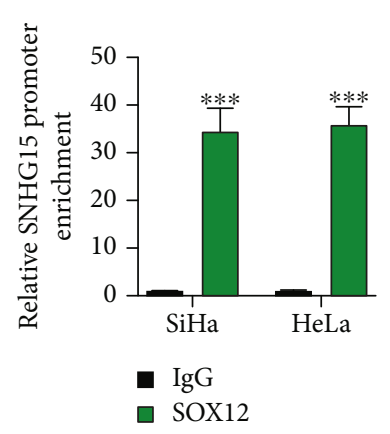

(f)

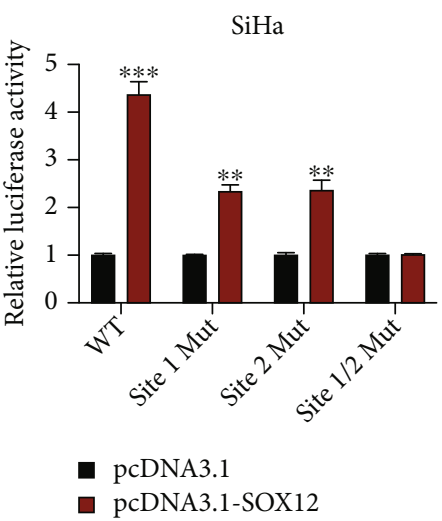

(g)

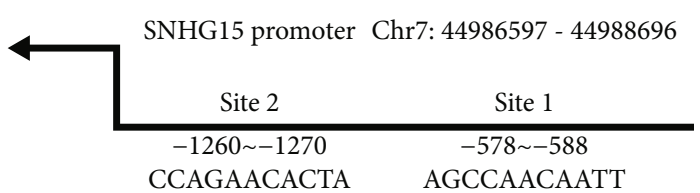

(e)

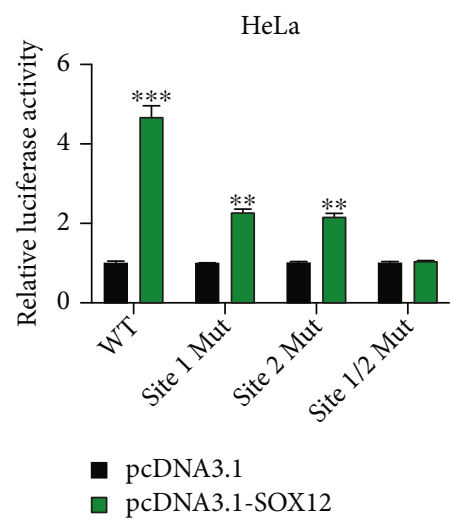

(h)

FIGURE 3: lncRNA SNHG15 expression in cervical cancer is transcriptionally regulated by SOX12. The upstream regulator of lncRNA SNHG15, predicted using the JASPAR (http://jaspar.genereg.net/) database. (a, b) SiHa and HeLa cells stably transfected with siRNAs; lncRNA SNHG15 expression, measured by qRT-PCR. (c) SiHa and HeLa cells transfected with pcDNA3.1-SOX12 and its normal control; lncRNA SNHG15 expression was assessed by qRT-PCR. (d) Binding motif of SOX12 predicted by JASPAR. (e) Putative binding sites for the lncRNA SNHG15 promoter, determined using JASPAR. (f) Binding possibility between SOX12 and the lncRNA SNHG15 promoter, assessed by ChIP assay. (g, h) Interaction between SOX12 and the lncRNA SNHG15 promoter in (g) SiHa and (h) HeLa cells, verified using the luciferase reporter gene assay. Data are presented as mean $\pm \mathrm{SD} ;{ }^{* *} p<0.01 ;{ }^{* * *} p<0.001$.

the miR-4735-3p inhibitor (Figures 5(h) and 5(i)). Transwell migration assay results suggested that CC cell migration was suppressed by the miR-4735 mimic but aggravated by the miR-4735-3p inhibitor (Figures 5(j) and 5(k), Supplementary Materials Figure S2C-S2D). For the same occurrence, the chemoresistance of CC cells to cisplatin was reduced by the miR-4735-3p mimic but aggravated by the miR-4735-3p inhibitor (Figures 5(l)-5(o)). Our results suggested that miR-4735-3p acted as a tumor suppressor in CC progression.

3.6. miR-4735-3p Targets to HIF1a. A total of 13 mRNA targets of miR-4735-3p were found by bioinformatics analysis
(Figure 6(a)). We then found that HIFla expression was decreased in miR-4735-3p overexpressed CC cells (Figures 6(b) and 6(c)) and increased in miR-4735-3p knockdown CC cells (Figure 6(d)), indicating that miR4735-3p could be a potential target to HIF1a. The binding sites between miR-4735-3p and HIFla were then obtained as indicated (Figure 6(e)). Data from the RNA pull-down assay showed that HIFla was noticeably enriched in biotinylated miR-4735-3p probes in CC cells (Figures 6(f) and $6(\mathrm{~g}))$. Meanwhile, luciferase reporter gene assay results confirmed that miR-4735-3p directly sponged to HIF1a in CC cells (Figures 6(h) and 6(i)). The interaction between miR4735-3p and HIFla was verified. HIFla expression in CC 


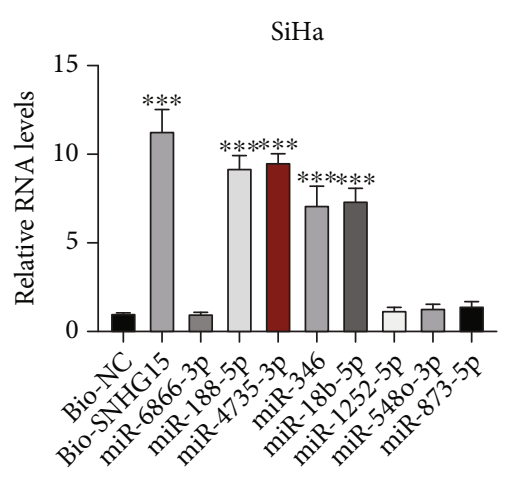

(a)

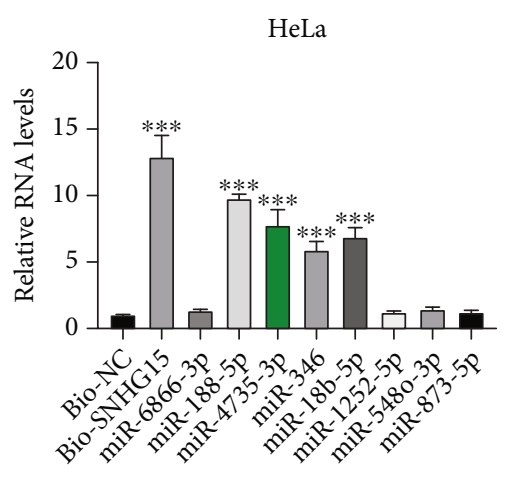

(b)

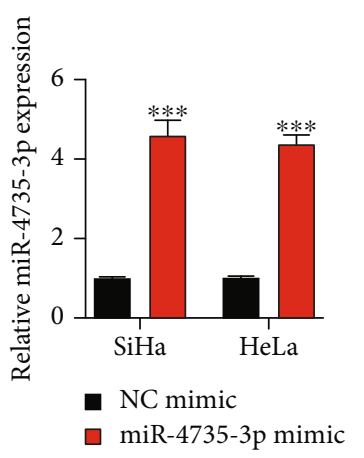

(e)

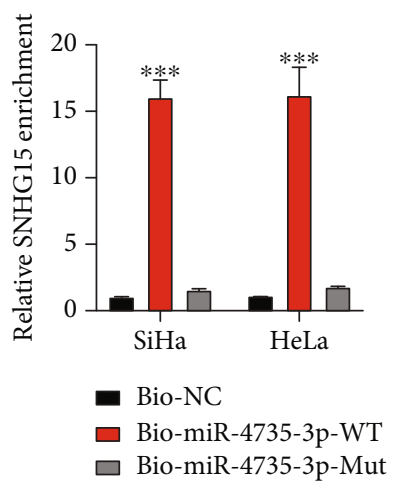

(c)

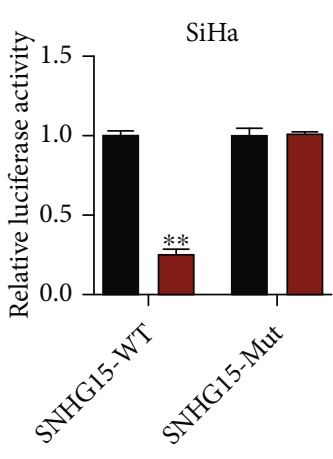

- NC mimic

a mIR-4735-3p mimic

(f)

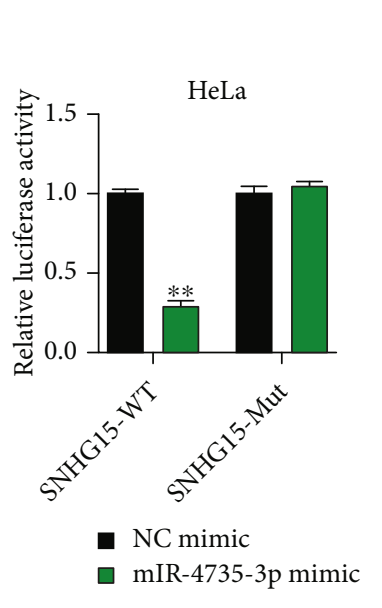

(g)

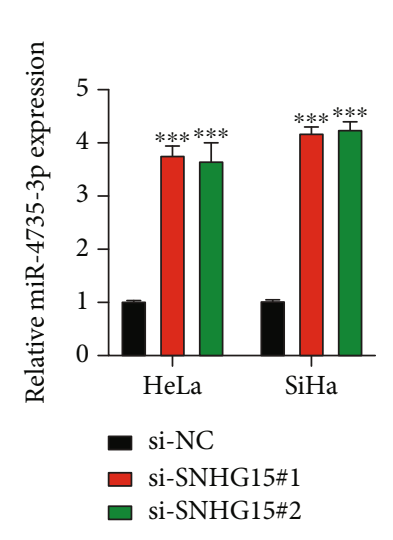

(h)

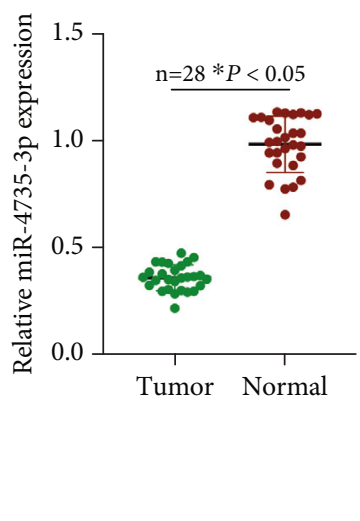

(i)

FiguRE 4: IncRNA SNHG15 sponges miR-4735-3p. Putative downstream targets for lncRNA SNHG15, predicted using the starBase (http:// starbase.sysu.edu.cn/) dataset (CLIP Data: strict stringency $(\geq 5)$ ). (a, b) Potential miRNA targets for lncRNA SNHG15 in SiHa and HeLa cells, evaluated by biotinylated RNA pull-down; expression levels of putative miRNA targets in biotinylated probe bounds were measured by qRT-PCR. (c) Expression levels of lncRNA SNHG15 in Bio-miR-4735-3p-WT or Bio-miR-4735-3p-Mut bounds, measured by qRTPCR. (d) Binding sites between lncRNA SNHG15 and miR-4735-3p, obtained using the starBase dataset. (e) Transfection efficiency of the miR-4735-3p mimic, evaluated by qRT-PCR. (f, g) Association between lncRNA SNHG15 and miR-4735-3p, assessed using the luciferase reporter gene assay in (f) $\mathrm{SiHa}$ and (g) HeLa. (h) miR-4735-3p expression in lncRNA SNHG15 knockdown cervical cancer (CC) cells, assessed by qRT-PCR. (i) miR-4735-3p expression in CC tumor samples $(n=28)$ and adjacent normal samples $(n=28)$, measured by qRT-PCR. Data are presented as mean \pm SD; ${ }^{*} p<0.5 ;{ }^{* *} p<0.01 ;{ }^{* * *} p<0.001$.

tissues was measured; HIFla expression in CC tumors was markedly higher than that in comparative normal tissues (Figures 6(j) and 6(k)).
3.7. IncRNA SNHG15 Regulates the Tumorigenesis and Chemoresistance of Cervical Cancer via the miR-4735-3p/ HIF1a Pathway. To further verify the molecular and cellular 


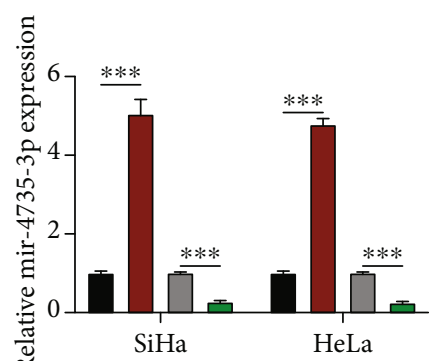

- NC mimic

- miR-4735-3p mimic

口 NC inhibitor

口 miR-4735-3p inhibitor

(a)

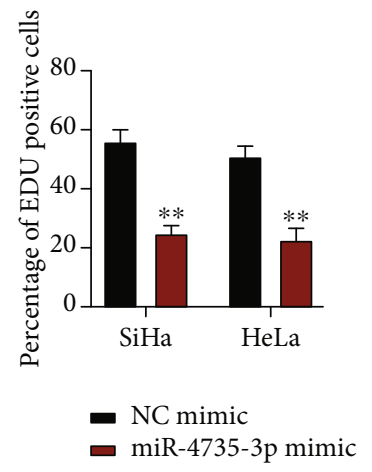

(e)

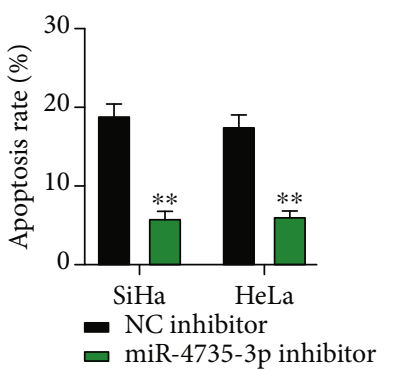

(i)

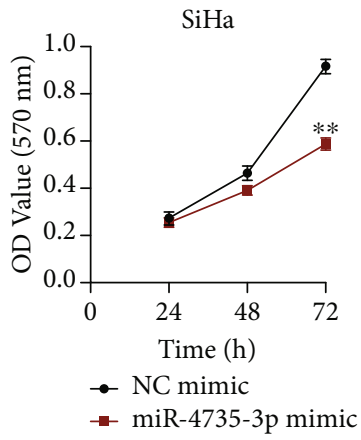

(b)

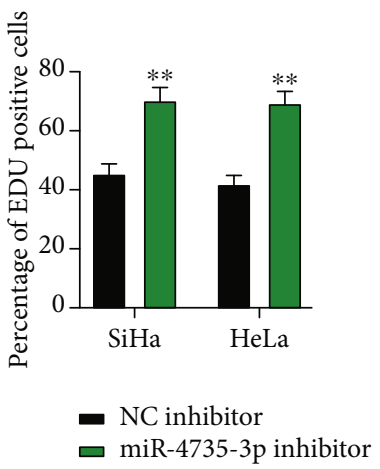

(f)

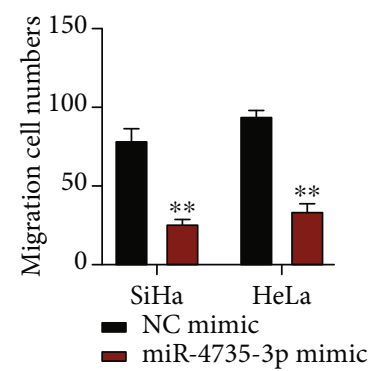

(j)
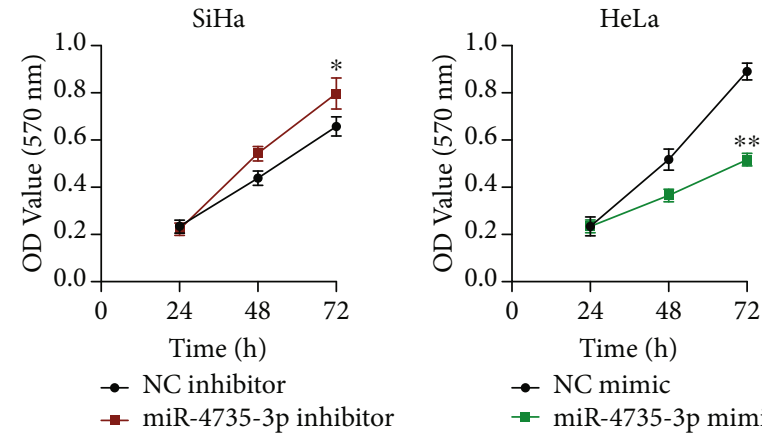

- NC mimic

$\rightarrow$ miR-4735-3p mimic

(c)

(d)
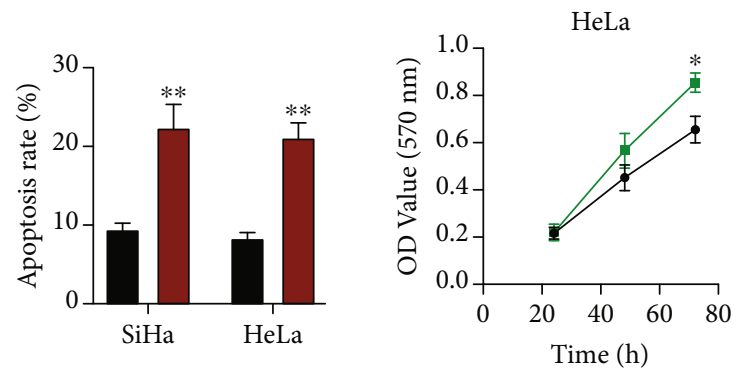

- NC mimic

- miR-4735-3p mimic

(g)
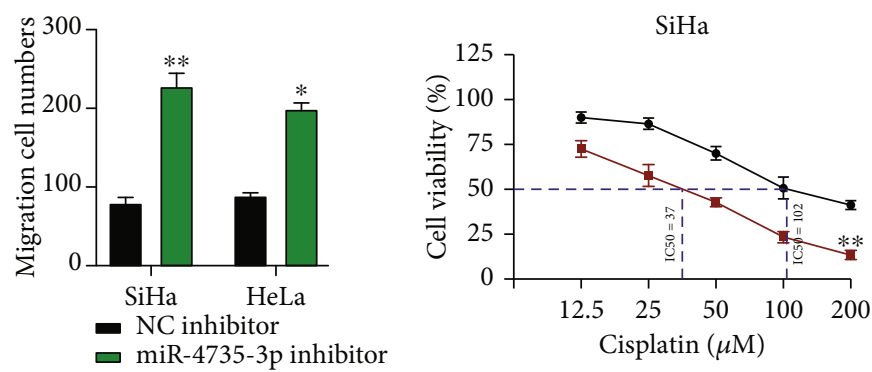

- NC mimic

$\rightarrow$ miR-4735-3p mimic

(1)

Figure 5: Continued. 


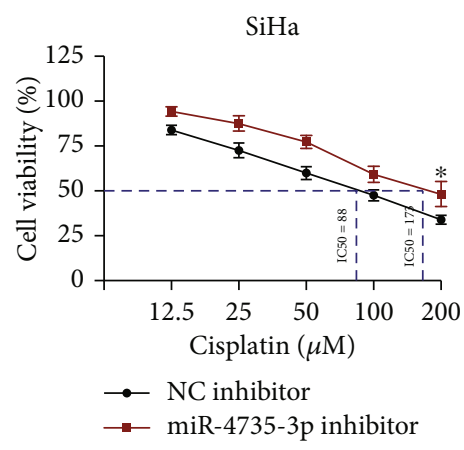

$(\mathrm{m})$

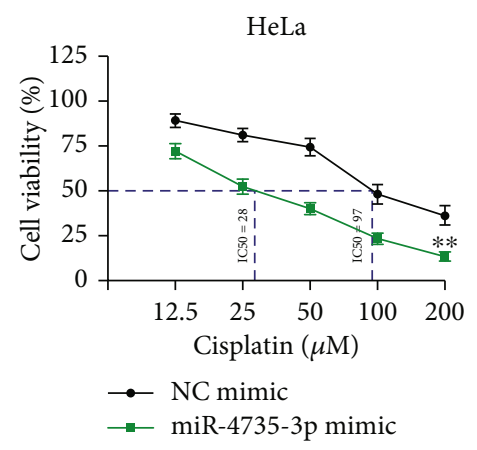

(n)

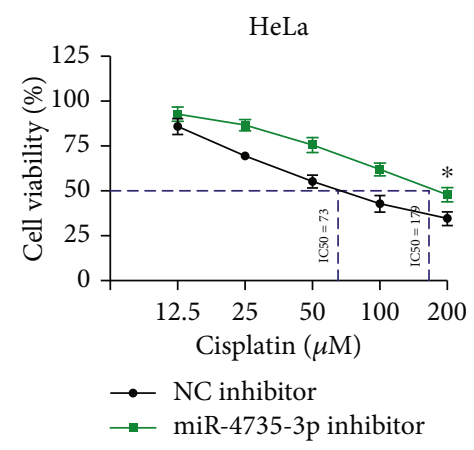

(o)

FIGURE 5: miR-4735-3p modulates tumorigenesis and chemoresistance in cervical cancer. (a) The gain- or loss-of-function of miR-4735-3p cell models was generated as indicated. (b-d) Cell proliferation of SiHa and HeLa cells upon miR-4735-3p dysregulation, detected using the MTT assay. (f, g) Cell proliferation levels of SiHa and HeLa cells upon miR-4735-3p (f) overexpression or (g) downregulation, evaluated using the EdU assay. (h, i) Cell apoptosis of SiHa and HeLa cells upon miR-4735-3p (h) overexpression or (i) downregulation, measured by flow cytometry. ( $\mathrm{j}, \mathrm{k}$ ) Cell migration levels of SiHa and HeLa cells upon miR-4735-3p (j) overexpression or (k) downregulation, assessed using the transwell migration assay. (1-o) Chemoresistance of SiHa and HeLa cells upon miR-4735-3p dysregulation, detected using the MTT assay. Data are presented as mean $\pm \mathrm{SD},{ }^{*} p<0.5 ;{ }^{* *} p<0.01 ;{ }^{* * *} p<0.001$.

functions of lncRNA SNHG15 in CC progression, we generated cell models by transfecting si-NC, si-SNHG15\#1, and si-SNHG15\#1+pcDNA3.1-HIF1a into SiHa and HeLa cells, and lncRNA SNHG15 or HIF1a expression was detected (Figures 7(a) and 7(b)). HIFla reversed the inhibitory effect of lncRNA SNHG15 knockdown on cell proliferation as detected using the MTT assay and the EdU assay (Figures 7(c)-7(e), Supplementary Materials Figure S3A). The cell apoptosis rate increased subsequent to IncRNA SNHG15 knockdown attenuated by HIF1a in CC cells (Figure 7(f)). Moreover, lncRNA SNHG15 knockdown suppressed cell migration level, and those occurrences were rescued by HIF1a in CC cells (Figure 7(g), Supplementary Materials Figure S3B). With regard to the chemoresistance of CC cells to cisplatin, lncRNA SNHG15 knockdown inhibited the IC50 value of SiHa and HeLa cells, but this occurrence was reversed by HIFla (Figures 7(h) and 7(i)). Our results showed that lncRNA SNHG15 modulated HIFla expression to regulate CC progression.

3.8. IncRNA SNHG15 Knockdown Suppresses CC Tumor Growth In Vivo. The aforementioned experiments demonstrated the function of lncRNA SNHG15 in vitro. We generated mouse models to examine the role of lncRNA SNHG15 in vivo. lncRNA SNHG15 knockdown cells $\left(10^{6}\right.$ per murine) were subcutaneously injected into the mice, and after 4 weeks, tumors were collected. The representative image of xenograft tumors is presented in Figure 8(a). The tumor volume and the end weight were significantly inhibited by lncRNA NHG15 knockdown (Figures 8(b) and 8(c)). Further, the expression levels of SNHG15, miR-4735-3p, and HIF1a in lncRNA SNHG15 knockdown tumor tissues were measured (Figures $8(\mathrm{~d})-8(\mathrm{~g})$ ), and $t$ and the trends were consistent with our in vitro experiments. The results obtained suggest that lncRNA SNHG15 regulated tumor growth in murine models via the miR-4735-3p/HIF1a axis.

\section{Discussion}

The role of the lncRNA/miRNA/mRNA network in various biological progression has been elucidated in recent decades. As a major cause of cancer initiation and progression, multiple gene mutations have drawn wide scientific interests, and considerable effort has been exerted to elucidate the molecular mechanisms that contribute to tumorigenesis via the lncRNA/miRNA/mRNA network, such as bladder cancer, hepatocellular carcinoma, osteosarcoma, gastric cancer, oral cancer, and CC [21-26]. IncRNA-CTS regulates CC progression via the miR-505/ZEB2 axis [27], IncRNA TP73-AS1 modulates CC development via the miR-3293p/ARF1 axis [28], IncRNA CAR10 promotes CC progression via the miR-125b-5p/PDPK1 pathway [29], and lncRNA DANCR aggravates CC development via the miR335-5p/ROCK1 axis [30]. Despite the accumulating reports on the lncRNA/miRNA/mRNA network globally, the main mechanism underlying gene dysregulation in cancer development has yet to be clarified.

In this study, we clarified elucidated that lncRNA SNHG15 was highly expressed in CC tissues and cells. Downregulated lncRNA SNHG15 attenuated tumorigenic properties in vitro and CC tumor growth in vivo. IncRNA SNHG15 dysregulation also influenced the chemoresistance of CC cells to cisplatin. Cisplatin belongs to the alkylating antineoplastic agent and is used in the clinical management of several malignancies, including CC [31-36]. With the wide clinical application of cisplatin, its side effects are been revealed, such as nausea, vomiting, kidney damage, hearing loss, and cell chemoresistance [37]. Understanding the molecular mechanisms underlying chemoresistance, among its side effects, is crucial to improving the therapy efficiency of cisplatin. The results of this study indicate that lncRNA SNHG15 downregulation inhibited the IC50 value of CC cells upon cisplatin treatment.

Further, for the upstream regulator of lncRNA SNHG15, we found that lncRNA SNHG15 expression in CC cells was 


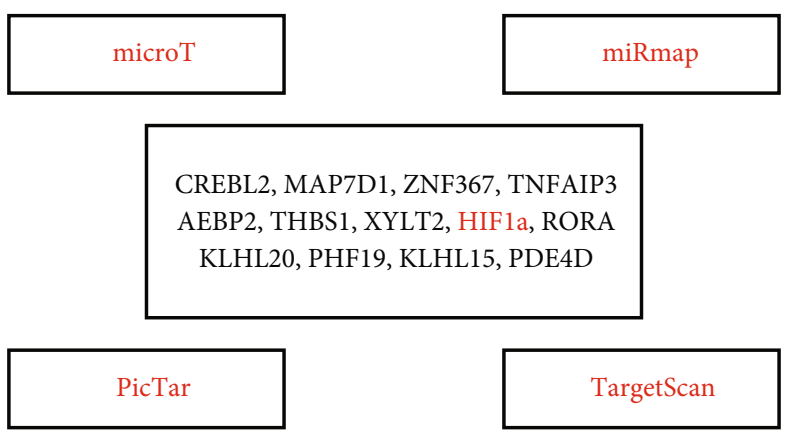

CLIP Data: strict stringency ( $>=5)$, and AgoExpNum $>=20$

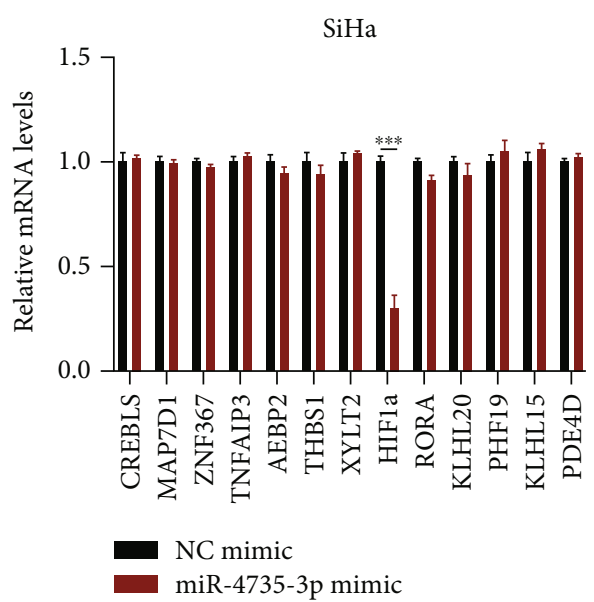

(b)

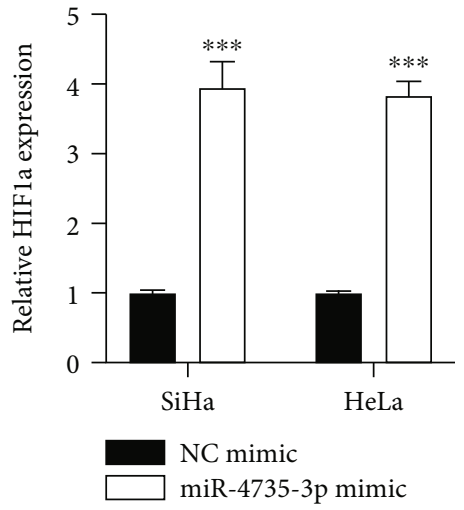

(d)

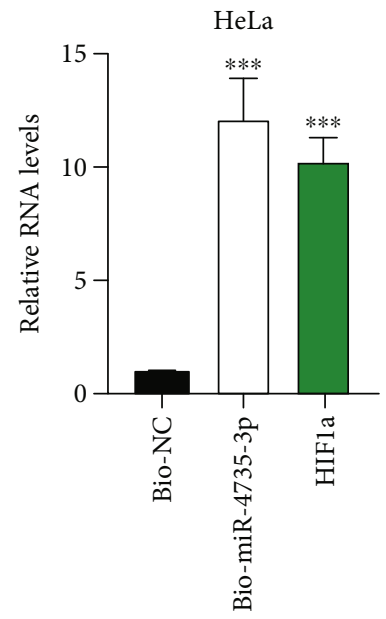

(f)

Figure 6: Continued 


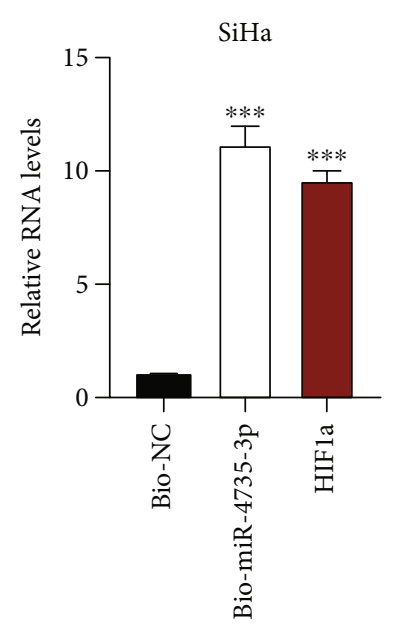

(g)

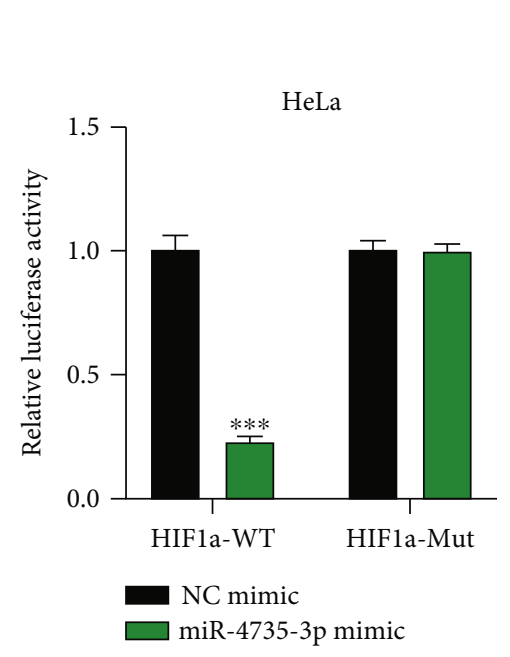

(i)

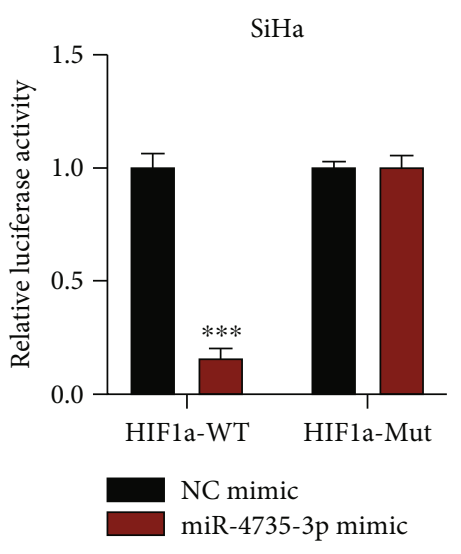

(h)

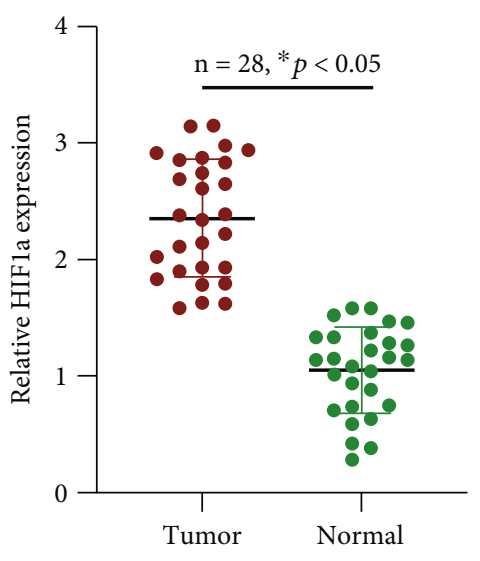

(j)

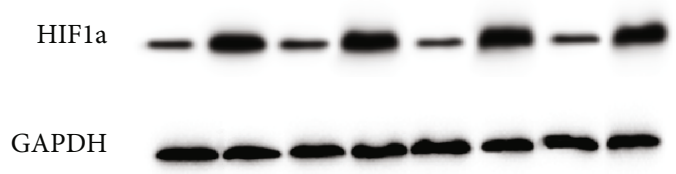

(k)

Figure 6: miR-4735-3p targets to HIF1a. (a) Putative downstream targets for miR-4735-3p, predicted by microT (http://www.microrna.gr/ microT), miRmap (http://mirnamap.mbc.nctu.edu.tw/), PicTar (http://www.pictar.org/), and TargetScan (http://www.targetscan.org/) with CLIP Data: strict stringency $(\geq 5)$ and AgoExpNum $\geq 20$. (b, c) Putative mRNA targets in (b) SiHa and (c) HeLa cells upon miR-4735-3p overexpression were measured by qRT-PCR. (d) HIF1a expression in cervical cancer (CC) cells upon miR-4735-3p downregulation, measured by qRT-PCR. (e) Binding sites between HIF1a and miR-4735-3p. (f, g) Association between miR-4735-3p and HIF1a in (f) $\mathrm{SiHa}$ and (g) HeLa cells, evaluated by ChIP assay; analysis of results was by qRT-PCR. (h, i) Interaction between HIF1a and miR-4735$3 \mathrm{p}$, assessed using the luciferase reporter gene assay in (h) SiHa and (i) HeLa. (j) HIF1a expression in CC tumor samples ( $n=28)$ and adjacent normal samples $(n=28)$, measured by qRT-PCR. (k) HIF1a expression in 4 randomly chosen pairs of CC tissues, detected by Western blot. Data are presented as mean $\pm \mathrm{SD} ;{ }^{*} p<0.5 ;{ }^{* * *} p<0.001$.

transcriptionally regulated by SOX12. For the downstream factors of lncRNA SNHG15, our results demonstrated that lncRNA SNHG15 promoted HIF1a expression via sponging of miR-4735-3p in CC cells. Our biological experiments revealed that IncRNA SNHG15 promotes the tumorigenic properties and chemoresistance of CC cells via the miR-4735-3p/HIF1a axis. Meanwhile, this study has several limitations. First, more human samples are required to confirm the clinical significance of lncRNA SNHG15 in CC tumors. Second, a larger scale of samples than the one used in this study is needed to examine the correlation of expression between lncRNA SNHG15, miR4735-3p, and HIF1a in CC tumors. Finally, the specific molecular relation between HIF1a and CC cellular progression requires an in-depth investigation, such as determining the involved pathway. 


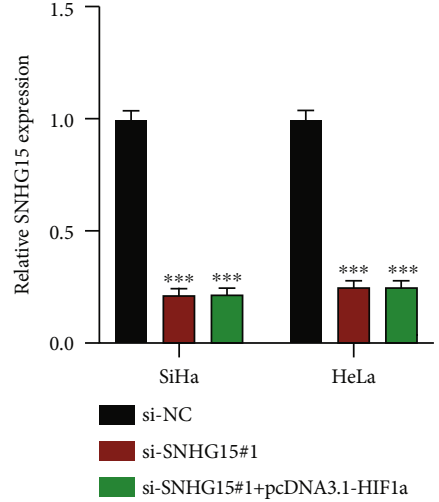

(a)

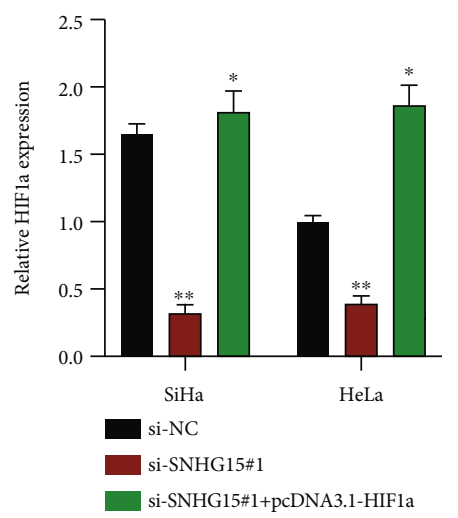

(b)

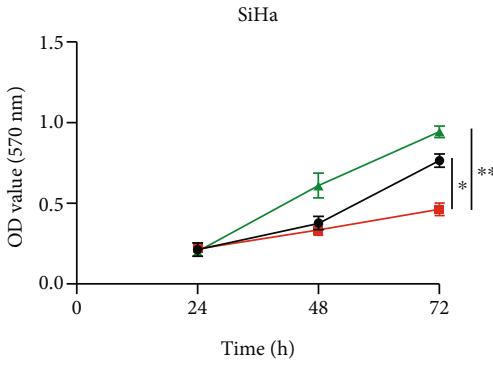

$\rightarrow$ si-NC

- si-SNHG15\#1

- si-SNHG15\#1+pcDNA3.1-HIFla

(c)

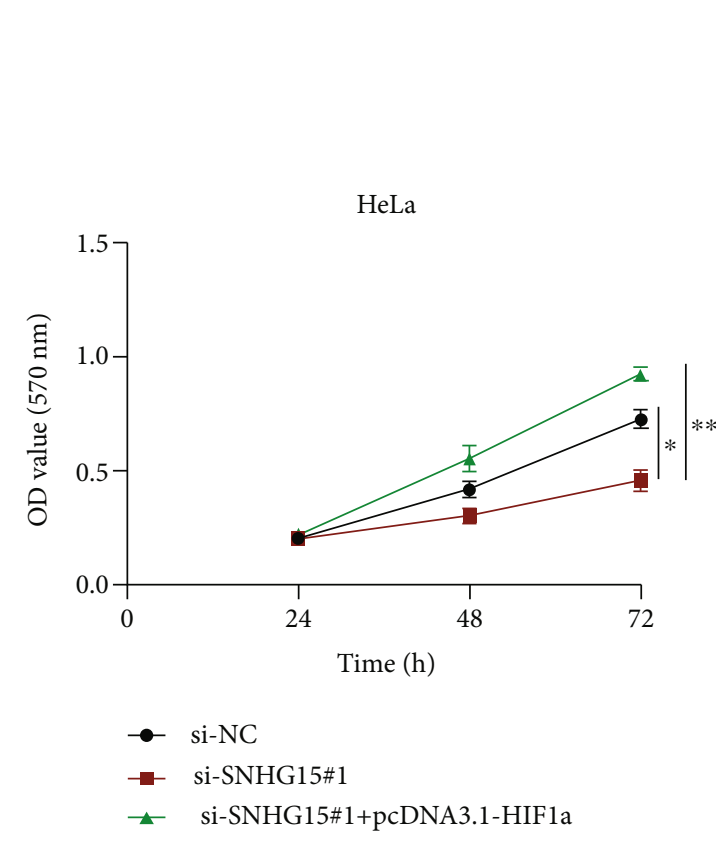

(d)

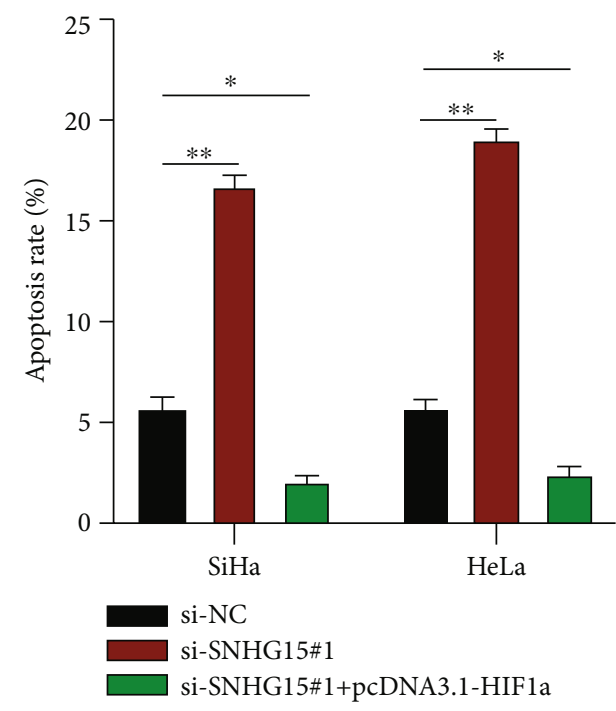

(f)

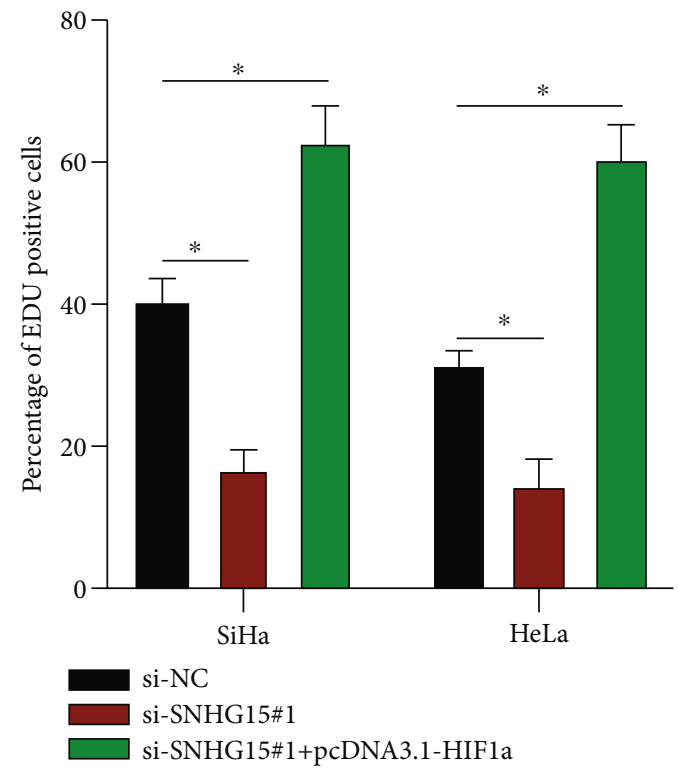

(e)

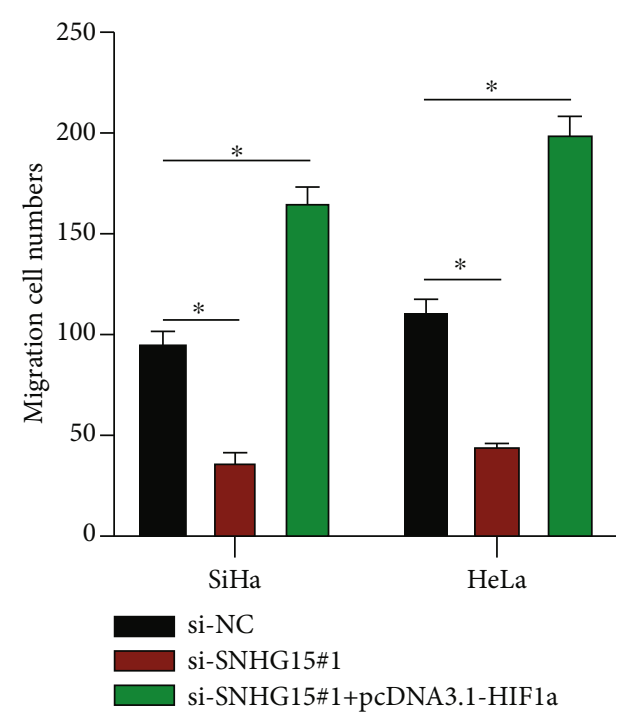

(g)

Figure 7: Continued. 
$\mathrm{SiHa}$

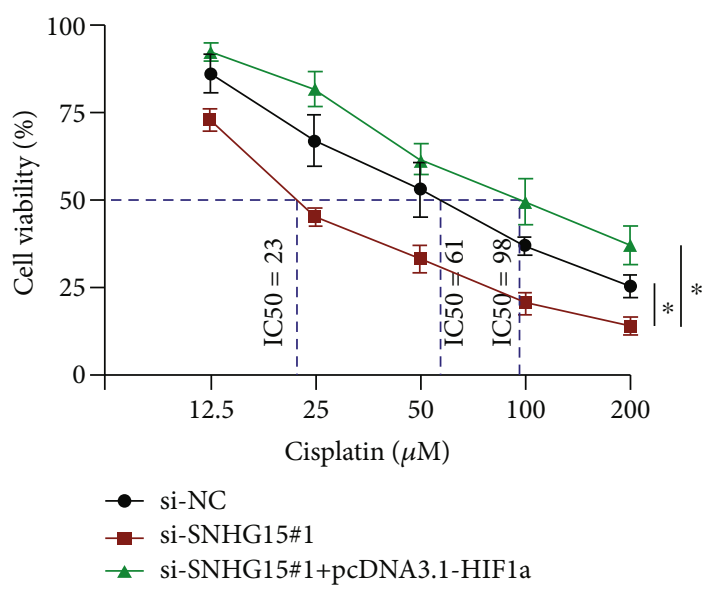

(h)

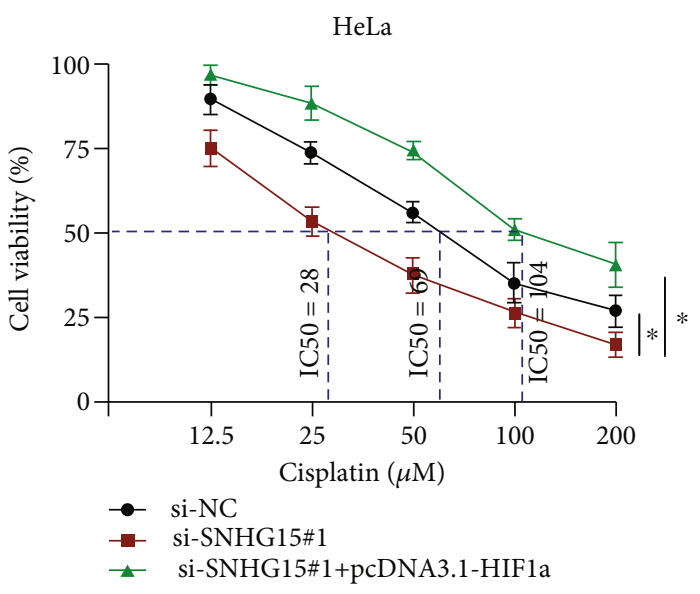

(i)

FIGURE 7: lncRNA SNHG15 regulates the tumorigenesis and chemoresistance of cervical cancer (CC) via the miR-4735-3p/HIF1a pathway. (a, b) Gain- or loss-of-function cell models were generated as indicated; assessment of transfection efficiencies. (c-e) Cell proliferation detected using the (c, d) MTT assay and the (e) EdU assay, respectively. (f) Cell apoptosis rate evaluated by flow cytometry. (g) Cell migration level assessed using the transwell migration assay. ( $\mathrm{h}$, i) $\mathrm{SiHa}$ and HeLa cells were treated with cisplatin at varying concentrations; the chemoresistance of CC cells was determined using the MTT assay. Data are presented as mean $\pm \mathrm{SD} ;{ }^{*} p<0.5 ;{ }^{* *} p<0.01 ;{ }^{* * *} p<0.001$.

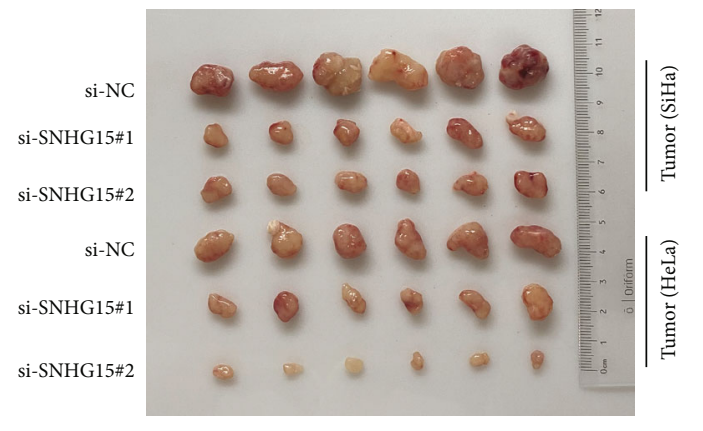

(a)

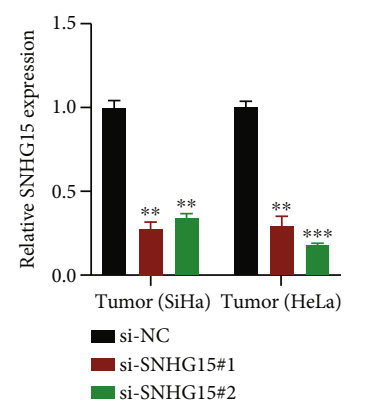

(d)

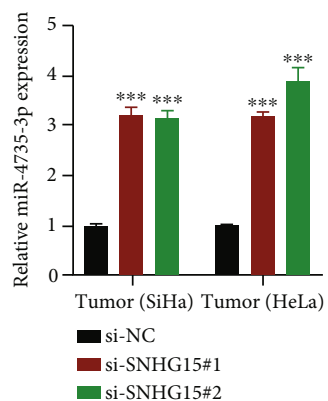

(e)

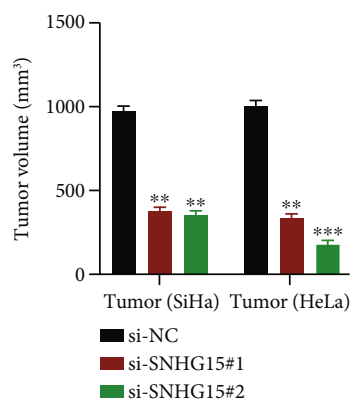

(b)

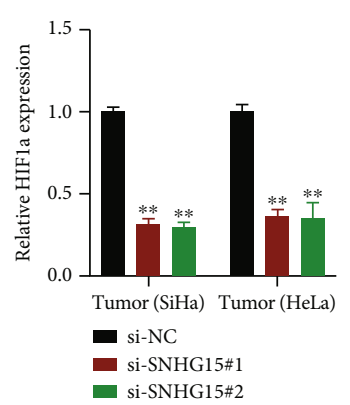

(f)

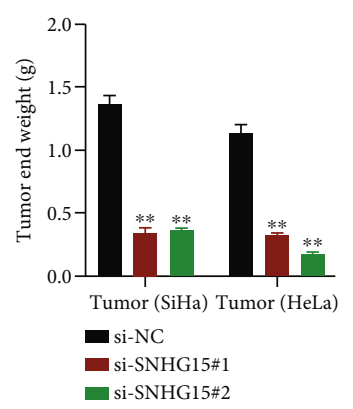

(c)

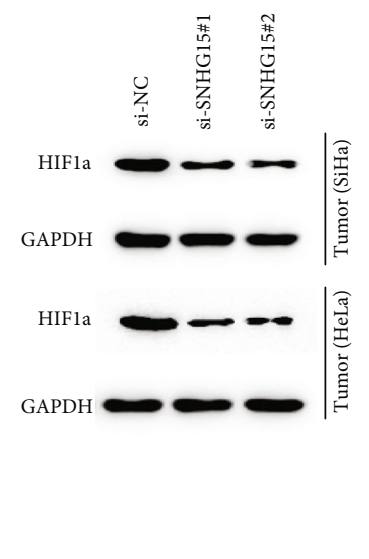

(g)

Figure 8: lncRNA SNHG15 knockdown suppresses tumor growth in cervical cancer in vivo. SiHa and HeLa cells stably transfected with siNC, si-SNHG15\#1, and si-SNHG15\#2 were subcutaneously injected into the murine models ( $10^{6}$ cells/tumor: $\left.n=6\right)$. (a) Representative image of xenograft tumors. (b) Tumor volume was recorded and calculated. (c) Tumor end weight was recorded. (d) lncRNA SNHG15 expression in tumors, measured by qRT-PCR. (e) miR-4735-3p expression determined by qRT-PCR. (f, g) HIF1a expression in resected tumor tissues, measured by qRT-PCR and Western blot analysis. Data are presented as mean \pm SD; ${ }^{* *} p<0.01 ;{ }^{* * *} p<0.001$. 


\section{Conclusion}

In summary, our study elucidates the expression and biological function of lncRNA SNHG15 in CC. This study also indicates that SOX12 could transcriptionally regulate lncRNA SNHG15 expression in CC cells. lncRNA SNHG15 promotes CC progression by regulating the miR-4735-3p/ HIFla axis, which could be a potential target for the diagnosis of CC or clinical intervention for the disease in the future.

\section{Data Availability}

The research data used to support the findings of this study are included within the article.

\section{Ethical Approval}

The studies involving human participants were reviewed and approved by the Medical Research Ethics Committee of Xiangyang Central Hospital.

\section{Consent}

Written informed consent for participation was not required for this study in accordance with the national legislation and the institutional requirements.

\section{Conflicts of Interest}

The authors declare that the research was conducted in the absence of any commercial or financial relationships that could be construed as a potential conflict of interest.

\section{Authors' Contributions}

HX and LL contributed to the study concept, design, administrative, and study supervision. JY, MY, and HL contributed to the acquisition of data, analysis, and interpretation of data. HX, XQ, YX, and LL contributed to the drafting of the manuscript and critical revision of the manuscript for important intellectual content. MZ, XM, and XQ contributed to the statistical analysis and technical and material support. All authors have read and approved the manuscript. Jiang Yang, Mei Yang, and Huabing Lv are considered as co-first authors.

\section{Acknowledgments}

This work was supported by the National Natural Science Foundation of China (81972449), the Hubei Provincial Natural Science Foundation of China (2019CFA016), Hubei Province's Outstanding Medical Academic Leader Program, the Research Foundation of Health Commission of Hubei Province, China (WJ2019M069), and the Scientific Research Ability Cultivation Fund of Hubei University of Arts and Science (2021kpgj06).

\section{Supplementary Materials}

Figure S1: lncRNA SNHG15 overexpression aggravates CC tumorigenesis and chemoresistance in vitro. (A) lncRNA SNHG15 level was measured by qRT-PCR in CC cells. (B, C) Cell proliferation was detected by MTT assay in $\mathrm{SiHa}$ (B) and HeLa cells (C). (D, E): EdU assay was applied to evaluate cell viabilities in SiHa and HeLa cells (D), and results were analyzed as indicated (E). (F, G) Cell apoptosis rate was measured by flow cytometer assay (F), and results were calculated as indicated $(\mathrm{G})$. (H, I) Transwell migration experiment was performed to assess cell migration level $(\mathrm{H})$, and statistical analysis was presented (I). (J, K) SiHa (J) and $\mathrm{HeLa}(\mathrm{K})$ cells were treated with cisplatin at various concentrations as indicated, and MTT assay was applied to evaluate the chemoresistance of CC cells. Data were presented as mean $\pm \mathrm{SD} ;{ }^{* *} p<0.01$ and ${ }^{* * *} p<0.001$. Figure S2: (A, B) EdU assay was applied to evaluate cell proliferation of $\mathrm{SiHa}$ and HeLa cells upon miR-4735-3p overexpression (A) or downregulation (B). (C, D) Transwell migration assay was conducted to assess cell migration level of $\mathrm{SiHa}$ and HeLa cells upon miR-4735-3p overexpression (C) or downregulation (D). Figure S3: (A) Cell proliferation was detected by EdU assay as indicated. (B) Cell migration level was assessed by transwell migration assay. (Supplementary Materials)

\section{References}

[1] C. de Martel, M. Plummer, J. Vignat, and S. Franceschi, "Worldwide burden of cancer attributable to HPV by site, country and HPV type," International Journal of Cancer, vol. 141, no. 4, pp. 664-670, 2017.

[2] N. C. Nkfusai, S. N. Cumber, T. Williams et al., "Cervical cancer in the Bamenda regional hospital, north west region of Cameroon: a retrospective study," The Pan African Medical Journal, vol. 32, p. 90, 2019.

[3] W. Hsu, L. Liu, X. Chen, Y. Zhang, and W. Zhu, "LncRNA CASC11 promotes the cervical cancer progression by activating Wnt/beta-catenin signaling pathway," Biological Research, vol. 52, no. 1, p. 33, 2019.

[4] S. Park, J. Kim, K. Eom et al., "microRNA-944 overexpression is a biomarker for poor prognosis of advanced cervical cancer," BMC Cancer, vol. 19, no. 1, p. 419, 2019.

[5] C. P. Ponting, P. L. Oliver, and W. Reik, "Evolution and functions of long noncoding RNAs," Cell, vol. 136, no. 4, pp. 629641, 2009.

[6] J. Q. Sheng, M. R. Wang, D. Fang et al., "LncRNA NBR2 inhibits tumorigenesis by regulating autophagy in hepatocellular carcinoma," Biomedicine \& Pharmacotherapy, vol. 133, article 111023, 2021.

[7] M. Zhang, N. Wang, P. Song et al., "LncRNA GATA3-AS1 facilitates tumour progression and immune escape in triple-negative breast cancer through destabilization of GATA3 but stabilization of PD-L1," Cell Proliferation, vol. 53, no. 9, article e12855, 2020.

[8] L. Yang, X. Peng, Y. Li et al., "Long non-coding RNA HOTAIR promotes exosome secretion by regulating RAB35 and SNAP23 in hepatocellular carcinoma," Molecular Cancer, vol. 18, no. 1, p. 78, 2019.

[9] M. Li, B. Yin, M. Chen et al., "Downregulation of the lncRNA ASB16-AS1 decreases LARP1 expression and promotes clear 
cell renal cell carcinoma progression via $\mathrm{miR}-185-5 \mathrm{p} / \mathrm{miR}-$ 214-3p," Frontiers in Oncology, vol. 10, article 617105, 2021.

[10] H. Tani and M. Torimura, "Identification of short-lived long non-coding RNAs as surrogate indicators for chemical stress response," Biochemical and Biophysical Research Communications, vol. 439, no. 4, pp. 547-551, 2013.

[11] H. Tani, S. Okuda, K. Nakamura, M. Aoki, and T. Umemura, "Short-lived long non-coding RNAs as surrogate indicators for chemical exposure and LINC00152 and MALAT1 modulate their neighboring genes," PLoS One, vol. 12, no. 7, article e0181628, 2017.

[12] Q. Kong and M. Qiu, "Long noncoding RNA SNHG15 promotes human breast cancer proliferation, migration and invasion by sponging miR-211-3p," Biochemical and Biophysical Research Communications, vol. 495, no. 2, pp. 1594-1600, 2018.

[13] H. Jiang, T. Li, Y. Qu et al., "Long non-coding RNA SNHG15 interacts with and stabilizes transcription factor Slug and promotes colon cancer progression," Cancer Letters, vol. 425, pp. 78-87, 2018.

[14] S. X. Chen, J. F. Yin, B. C. Lin et al., "Upregulated expression of long noncoding RNA SNHG15 promotes cell proliferation and invasion through regulates MMP2/MMP9 in patients with GC," Tumour Biology, vol. 37, no. 5, pp. 6801-6812, 2016.

[15] J. H. Zhang, H. W. Wei, and H. G. Yang, "Long noncoding RNA SNHG15, a potential prognostic biomarker for hepatocellular carcinoma," European Review for Medical and Pharmacological Sciences, vol. 20, no. 9, pp. 1720-1724, 2016.

[16] B. Jin, H. Jin, H. B. Wu, J. J. Xu, and B. Li, "Long non-coding RNA SNHG15 promotes CDK14 expression via miR-486 to accelerate non-small cell lung cancer cells progression and metastasis," Journal of Cellular Physiology, vol. 233, no. 9, pp. 7164-7172, 2018.

[17] D. Chen, Z. Zhang, X. Lu, and X. Yang, "Long non-coding RNA SNHG15 regulates cardiomyocyte apoptosis after hypoxia/reperfusion injury via modulating miR-188-5p/PTEN axis," Archives of Physiology and Biochemistry, pp. 1-8, 2020.

[18] T. Wang, D. Liang, and H. Yang, "SNHG15 facilitated malignant behaviors of oral squamous cell carcinoma through targeting miR-188-5p/DAAM1," Journal of Oral Pathology \& Medicine, vol. 50, no. 7, pp. 681-691, 2021.

[19] B. Xiong, Y. Wang, Y. Liu, C. Wu, and J. Chen, "The circulating LncRNA SNHG15/miR-346 axis may be a potential biomarker of cardiomyocyte apoptosis during myocardial ischemia/reperfusion injury," International Journal of Cardiology, vol. 334, p. 30, 2021.

[20] W. Chen, L. Huang, J. Liang, Y. Ye, S. Yu, and Y. Zhang, "Long noncoding RNA small nucleolar RNA host gene 15 deteriorates liver cancer via microRNA-18b-5p/LIM-only 4 axis," IUBMB Life, vol. 73, no. 2, pp. 349-361, 2021.

[21] M. Huang, Y. Long, Y. Jin et al., "Comprehensive analysis of the IncRNA-miRNA-mRNA regulatory network for bladder cancer," Translational Andrology and Urology, vol. 10, no. 3, pp. 1286-1301, 2021.

[22] L. Zhang, H. Tao, J. Li, E. Zhang, H. Liang, and B. Zhang, "Comprehensive analysis of the competing endogenous circRNA-lncRNA-miRNA-mRNA network and identification of a novel potential biomarker for hepatocellular carcinoma," Aging (Albany NY), vol. 13, no. 12, article 203056, pp. 15990-16008, 2021.
[23] J. Y. Wang, Y. Yang, Y. Ma et al., "Potential regulatory role of lncRNA-miRNA-mRNA axis in osteosarcoma," Biomedicine \& Pharmacotherapy, vol. 121, article 109627, 2020.

[24] J. Wang, Y. Ding, Y. Wu, and X. Wang, "Identification of the complex regulatory relationships related to gastric cancer from lncRNA-miRNA-mRNA network," Journal of Cellular Biochemistry, vol. 121, no. 1, pp. 876-887, 2020.

[25] J. Yin, X. Zeng, Z. Ai, M. Yu, Y.'. Wu, and S. Li, “Construction and analysis of a lncRNA-miRNA-mRNA network based on competitive endogenous RNA reveal functional lncRNAs in oral cancer," BMC Medical Genomics, vol. 13, no. 1, p. 84, 2020.

[26] J. Wang and C. Zhang, "Identification and validation of potential mRNA- microRNA- long-noncoding RNA (mRNAmiRNA-lncRNA) prognostic signature for cervical cancer," Bioengineered, vol. 12, no. 1, pp. 898-913, 2021.

[27] S. Feng, W. Liu, X. Bai et al., "LncRNA-CTS promotes metastasis and epithelial-to-mesenchymal transition through regulating miR-505/ZEB2 axis in cervical cancer," Cancer Letters, vol. 465, pp. 105-117, 2019.

[28] J. Xu and J. Zhang, "LncRNA TP73-AS1 is a novel regulator in cervical cancer via miR-329-3p/ARF1 axis," Journal of Cellular Biochemistry, vol. 121, no. 1, pp. 344-352, 2020.

[29] T. Hu, Q. Zhang, and L. Gao, "LncRNA CAR10 upregulates PDPK1 to promote cervical cancer development by sponging miR-125b-5p," BioMed Research International, vol. 2020, Article ID 4351671, 16 pages, 2020.

[30] H. Liang, C. Zhang, H. Guan, J. Liu, and Y. Cui, "LncRNA DANCR promotes cervical cancer progression by upregulating ROCK1 via sponging miR-335-5p," Journal of Cellular Physiology, vol. 234, no. 5, pp. 7266-7278, 2019.

[31] S. Dasari and P. Bernard Tchounwou, "Cisplatin in cancer therapy: molecular mechanisms of action," European Journal of Pharmacology, vol. 740, pp. 364-378, 2014.

[32] J. P. Pignon, H. Tribodet, G. V. Scagliotti et al., "Lung adjuvant cisplatin evaluation: a pooled analysis by the LACE Collaborative Group," Journal of Clinical Oncology, vol. 26, no. 21, pp. 3552-3559, 2008.

[33] X. C. Hu, J. Zhang, B. H. Xu et al., "Cisplatin plus gemcitabine versus paclitaxel plus gemcitabine as first-line therapy for metastatic triple-negative breast cancer (CBCSG006): a randomised, open-label, multicentre, phase 3 trial," The Lancet Oncology, vol. 16, no. 4, pp. 436-446, 2015.

[34] R. Agarwal and S. B. Kaye, "Ovarian cancer: strategies for overcoming resistance to chemotherapy," Nature Reviews. Cancer, vol. 3, no. 7, pp. 502-516, 2003.

[35] J. S. Cooper, T. F. Pajak, A. A. Forastiere et al., "Postoperative concurrent radiotherapy and chemotherapy for high-risk squamous-cell carcinoma of the head and neck," The New England Journal of Medicine, vol. 350, no. 19, pp. 1937-1944, 2004.

[36] H. Zhu, H. Zhu, H. Luo, W. Zhang, Z. Shen, and X. Hu, "Molecular mechanisms of cisplatin resistance in cervical cancer," Drug Design, Development and Therapy, vol. 10, pp. 1885-1895, 2016.

[37] M. R. Trendowski, O. el Charif, P. C. Dinh Jr., L. B. Travis, and M. E. Dolan, "Genetic and modifiable risk factors contributing to cisplatin-induced toxicities," Clinical Cancer Research, vol. 25, no. 4, pp. 1147-1155, 2019. 